\title{
Vitamin C administration in the critically ill: a summary of recent meta-analyses
}

\author{
Anitra C. CarriD \\ This comment refers to the article available at https://doi.org/10.1186/s13054-018-2191-x.
}

Since 2018, there has been a dramatic upsurge in publications relating to the use of vitamin $\mathrm{C}$ in critically ill patients, particularly those suffering from sepsis [1]. This has primarily been in response to the well-publicized before-and-after study of Marik et al. [2], which indicated that intravenous administration of $6 \mathrm{~g} /$ day vitamin $C$ (in combination with thiamine and hydrocortisone) could improve the outcomes of patients with sepsis, including decreasing mortality. Over the past year, seven meta-analyses assessing the effects of vitamin $\mathrm{C}$ administration in critically ill patients have been published, with four appearing in the past 4 months alone (see Table 1 summary).

The most recently published and largest metaanalysis included 44 randomized controlled trials (16 intensive care and 28 cardiac surgery) [9]. Although meta-analyses that include a larger number of studies have increased power, they run the risk of comparing disparate studies. This is particularly the case with vitamin C studies whereby the dose (milligrams vs grams), rout of administration (oral vs intravenous), duration (hours vs days), and disease (e.g., sepsis vs cardiac surgery) can have a large impact on outcomes $[10,11]$. Therefore, appropriate subgroup analyses should be carried out, although this is currently challenging due to the low number of comparable studies.
All but one of the current meta-analyses have focused on mortality as a primary outcome, despite many of the included trials having relatively low numbers of patients. In most cases, no effect of intervention was observed on mortality, except in specific subgroup analyses (e.g., sepsis and higher dose intravenous vitamin $\mathrm{C}$ ). However, there have been few of these studies published to date, and even fewer of high methodological quality [6]. Other commonly assessed outcomes included ICU and hospital length of stay, duration of vasopressor support and mechanical ventilation, and acute kidney injury. Some of the meta-analyses showed decreases in several of these secondary outcomes, while others showed no effect, depending on the selection criteria used for study inclusion (Table 1).

There are currently over a dozen randomized controlled trials registered on clinicaltrials.gov that are assessing the effects of vitamin $\mathrm{C}$ administration in critically ill patients, particularly those with sepsis. One would hope that in the short term, no more meta-analyses appear every time another small study is published, but instead wait until some of the larger trials (such as VICTAS and LOVIT) have been completed. Otherwise, there may end up with more meta-analyses than published trials. 
Table 1 A summary of recent meta-analysis of vitamin C administration in critical care patients

\begin{tabular}{|c|c|c|c|c|c|}
\hline $\begin{array}{l}\text { Publication } \\
\text { details }\end{array}$ & Title & Selection criteria (PICO) & Included studies & Subgroup analysis & Findings \\
\hline $\begin{array}{l}\text { Putzu et al. } \\
\text { [3] } \\
\text { Crit Care } \\
\text { Med }\end{array}$ & $\begin{array}{l}\text { The effect of vitamin C on } \\
\text { clinical outcome in critically } \\
\text { ill patients: A systematic } \\
\text { review with meta-analysis } \\
\text { of randomised controlled } \\
\text { trials }\end{array}$ & $\begin{array}{l}\text { P_adult critically ill patients } \\
\text { I-vitC (any regimen) } \\
\text { C-placebo or no therapy } \\
\text { O-mortality, acute kidney } \\
\text { injury, supraventricular } \\
\text { arrhythmia, ventricular arrhythmia, } \\
\text { stroke, ICU LOS, hospital LOS }\end{array}$ & $\begin{array}{l}44 \text { RCTs: } \\
16 \text { in ICU setting }(n=2857) \\
28 \text { in cardiac surgery } \\
(n=3598)\end{array}$ & $\begin{array}{l}\text { Mixed ICU vs burns } \\
\text { vs sepsis/septic shock } \\
\text { vs acute pancreatitis } \\
\text { VitC alone vs enteral } \\
\text { vitC vs IV vitC vs IV vitC } \\
>5 \mathrm{~g}\end{array}$ & $\begin{array}{l}\text { ICU patients: } \\
\text { X mortality } \\
\text { X acute kidney } \\
\text { injury } \\
\text { X ICU or hospital } \\
\text { LOS } \\
\text { Cardiac surgery: } \\
\downarrow \text { postoperative } \\
\text { atrial fibrillation } \\
\downarrow \text { ICU and hospital } \\
\text { LOS }\end{array}$ \\
\hline $\begin{array}{l}\text { Wang et al. } \\
{[4]} \\
\text { Ann } \\
\text { Intensive } \\
\text { Care }\end{array}$ & $\begin{array}{l}\text { Effects of different ascorbic } \\
\text { acid doses on the mortality } \\
\text { of critically ill patients: a } \\
\text { meta-analysis }\end{array}$ & $\begin{array}{l}\text { P_critically ill patients } \\
\text { I-IV vitC (including } \\
\text { co-administration of antioxidants) } \\
\text { C- - placebo or no intervention } \\
\text { O-mortality, resuscitation fluid } \\
\text { requirement, urine output, acute } \\
\text { kidney injury, vasopressor } \\
\text { requirement, duration of } \\
\text { mechanical ventilation, ICU } \\
\text { and/or hospital LOS }\end{array}$ & $\begin{array}{l}12 \mathrm{RCT} \text {, quasi-RCT, } \\
\text { observational }(n=1210)\end{array}$ & $\begin{array}{l}\text { Low dose vs medium } \\
\text { dose vs high dose } \\
\text { Burn vs sepsis vs } \\
\text { others }\end{array}$ & $\begin{array}{l}\downarrow \text { mortality (doses of } \\
3-10 \mathrm{~g} / \text { day) } \\
X \text { morality }(<3 \mathrm{~g} / \\
\text { day or } \geq 10 \mathrm{~g} / \text { day) } \\
\downarrow \text { duration of } \\
\text { vasopressor support } \\
\downarrow \text { duration of } \\
\text { mechanical } \\
\text { ventilation } \\
X \text { acute kidney } \\
\text { injury } \\
\text { X ICU or hospital } \\
\text { LOS } \\
X \text { fluid requirement } \\
\text { X urine output }\end{array}$ \\
\hline $\begin{array}{l}\text { Hemila and } \\
\text { Chalker [5] }\end{array}$ & $\begin{array}{l}\text { Vitamin C can shorten the } \\
\text { length of stay in the ICU: A } \\
\text { meta-analysis }\end{array}$ & $\begin{array}{l}\text { P-ICU patients } \\
\text { I-vitC } \\
\text { C-placebo or none } \\
\text { O_ICU LOS, duration of } \\
\text { mechanical ventilation }\end{array}$ & $\begin{array}{l}18 \text { controlled trials } \\
(n=2004) \\
\text { including } 13 \text { cardiac surgery }\end{array}$ & $\begin{array}{l}\text { IV vs oral } \\
1-2 \text { days ICU vs } 3-5 \\
\text { days ICU } \\
>24 \mathrm{~h} \text { ventilation } \\
\text { vs }<24 \mathrm{~h} \text { ventilation }\end{array}$ & $\begin{array}{l}\downarrow I C U \text { LOS } \\
\downarrow \text { duration of } \\
\text { mechanical } \\
\text { ventilation }\end{array}$ \\
\hline $\begin{array}{l}\text { Langlois et } \\
\text { al. [6] } \\
\text { JPEN }\end{array}$ & $\begin{array}{l}\text { Vitamin C supplementation } \\
\text { in the critically ill: A } \\
\text { systematic review and } \\
\text { meta-analysis }\end{array}$ & $\begin{array}{l}\text { P_ICU patients } \\
\text { I-vitC (enteral or parenteral) } \\
\text { C-placebo or none } \\
\text { O_-mortality, incident infections, } \\
\text { ICU LOS, hospital LOS, duration } \\
\text { of mechanical ventilation }\end{array}$ & $\begin{array}{l}11 \text { RCTs } \\
9 \text { RCTs with mortality } \\
(n=1322)\end{array}$ & $\begin{array}{l}\text { Low dose vs high dose } \\
\text { Combined therapy vs } \\
\text { monotherapy } \\
\text { Oral/enteral vs } \\
\text { parenteral } \\
\text { Non-septic vs septic } \\
\text { Higher-quality trials vs } \\
\text { low-quality trials }\end{array}$ & $\begin{array}{l}\text { X mortality } \\
\downarrow \text { (trend) mortality } \\
\text { (IV high dose vitC } \\
\text { monotherapy) } \\
\text { X infections } \\
\text { X ICU or hospital } \\
\text { LOS } \\
\text { X duration of } \\
\text { mechanical } \\
\text { ventilation }\end{array}$ \\
\hline $\begin{array}{l}\text { Zhang and } \\
\text { Jativa [7] } \\
\text { SAGE Open } \\
\text { Med }\end{array}$ & $\begin{array}{l}\text { Vitamin C supplementation } \\
\text { in the critically ill: A } \\
\text { systematic review and } \\
\text { meta-analysis }\end{array}$ & $\begin{array}{l}\text { P_-critically ill adult patients } \\
\text { I-IV vitC } \\
\text { C- } \text { - placebo or no intervention } \\
\text { O_-mortality, duration of } \\
\text { mechanical ventilation, duration } \\
\text { of vasopressor support, fluid } \\
\text { requirements, urine output }\end{array}$ & $\begin{array}{l}4 \text { RCTs and } 1 \text { retrospective } \\
(n=142)\end{array}$ & & $\begin{array}{l}X \text { mortality } \\
\downarrow \text { need for } \\
\text { vasopressor support } \\
\downarrow \text { duration of } \\
\text { mechanical } \\
\text { ventilation } \\
\downarrow \text { (trend) fluid } \\
\text { requirements } \\
\uparrow \text { (trend) urine } \\
\text { output }\end{array}$ \\
\hline $\begin{array}{l}\mathrm{Li} \\
\text { Crit Care } \\
{[1]}\end{array}$ & $\begin{array}{l}\text { Evidence is stronger than } \\
\text { you think: a meta-analysis } \\
\text { of vitamin C use in patients } \\
\text { with sepsis }\end{array}$ & $\begin{array}{l}\text { P_-patients with sepsis } \\
\text { I-IV vitC } \\
\text { C-placebo or none } \\
\text { O_-mortality, ICU LOS, } \\
\text { vasopressor duration }\end{array}$ & 2 RCTs and 1 before-after & & $\begin{array}{l}\downarrow \text { mortality } \\
\text { X ICU LOS } \\
\downarrow \text { vasopressor } \\
\text { duration }\end{array}$ \\
\hline $\begin{array}{l}\text { Lin et al. [8] } \\
\text { Open J } \\
\text { Intern Med }\end{array}$ & $\begin{array}{l}\text { Adjuvant administration of } \\
\text { vitamin } C \text { improves } \\
\text { mortality of patients with } \\
\text { sepsis and septic shock: } A \\
\text { systems review and meta- } \\
\text { analysis }\end{array}$ & $\begin{array}{l}\text { P_-patients with septic } \\
\text { shock and severe sepsis } \\
\text { I_vitC } \\
\text { C_-placebo } \\
\text { O_-mortality }\end{array}$ & $\begin{array}{l}4 \text { RCTs and } 2 \text { retrospective } \\
\text { studies }(n=109)\end{array}$ & $\begin{array}{l}\text { RCT vs retrospective } \\
\text { High dose vs low dose }\end{array}$ & $\begin{array}{l}\text { X mortality } \\
\downarrow \text { mortality (doses of } \\
>50 \mathrm{mg} / \mathrm{kg} / \text { day) } \\
\text { X ICU LOS }\end{array}$ \\
\hline
\end{tabular}

ICU intensive care unit, IV intravenous, LOS length of stay, PICO patients, intervention, comparator, outcome, RCT randomized controlled trial, vitC vitamin C 


\section{Acknowledgements}

NA

\section{Author's contributions}

AC wrote the letter. The author read and approved the final manuscript.

\section{Funding}

AC is supported by a Health Research Council of New Zealand Sir Charles Hercus Health Research Fellowship.

\section{Availability of data and materials \\ NA}

\section{Ethics approval and consent to participate}

NA

\section{Consent for publication}

NA

\section{Competing interests}

The author declares that she has no competing interests.

Received: 20 June 2019 Accepted: 8 July 2019

Published online: 30 July 2019

\section{References}

1. Li J. Evidence is stronger than you think: a meta-analysis of vitamin C use in patients with sepsis. Crit Care. 2018;22(1):258.

2. Marik PE, Khangoora V, Rivera R, Hooper MH, Catravas J. Hydrocortisone, vitamin $C$, and thiamine for the treatment of severe sepsis and septic shock: a retrospective before-after study. Chest. 2017;151(6):1229-38.

3. Putzu A, Daems AM, Lopez-Delgado JC, Giordano VF, Landoni G. The effect of vitamin C on clinical outcome in critically ill patients: A systematic review with meta-analysis of randomized controlled trials. Crit Care Med. 2019;47(6):774-83.

4. Wang $Y$, Lin H, Lin BW, Lin JD. Effects of different ascorbic acid doses on the mortality of critically ill patients: a meta-analysis. Annals of intensive care. 2019;9(1):58.

5. Hemila $\mathrm{H}$, Chalker E. Vitamin C can shorten the length of stay in the ICU: A meta-analysis. Nutrients. 2019;11(4).

6. Langlois PL, Manzanares W, Adhikari NKJ, Lamontagne F, Stoppe C, Hill A, et al. Vitamin C supplementation in the critically ill: A systematic review and meta-analysis. JPEN Parenter Enteral Nutr. 2019.

7. Zhang M, Jativa DF. Vitamin C supplementation in the critically ill: A systematic review and meta-analysis. SAGE open medicine.2018;6: 2050312118807615.

8. Lin J, Li H, Wen Y, Zhang M. Adjuvant administration of vitamin C improves mortality of patients with sepsis and septic shock: A systems review and meta-analysis. Open J Intern Med 2018;8:146-59.

9. Hooper MH, Hager DN. Understanding vitamin C in critical illness: a focus on dose, route, and disease. Crit Care Med. 2019;47(6):867-9.

10. Carr AC. Duration of intravenous vitamin C therapy is a critical consideration. Crit Care Resuscitation. 2019;In press.

11. Carr AC. Vitamin C in pneumonia and sepsis. In: Vissers M, Chen Q, editors. Oxidative Stress and Disease. Boca Raton Taylor and Francis Group; 2019. In press.

\section{Publisher's Note}

Springer Nature remains neutral with regard to jurisdictional claims in published maps and institutional affiliations. 